\title{
Interleukin-18 Expression Increases in the Aorta and Plasma of Patients with Acute Aortic Dissection
}

\author{
Haiying Hu, ${ }^{1}$ Guangtai Zhang, ${ }^{2}$ Huanli Hu, ${ }^{3}$ Wenjing Liu, ${ }^{1}$ Jixiang Liu, ${ }^{1}$ Shuanli Xin, ${ }^{1}$ \\ Xiufeng Zhao, ${ }^{1}$ Liying Han, ${ }^{1}$ Liping Duan, ${ }^{1}$ Xinshun Huang, ${ }^{4}$ and Chao Chang ${ }^{1}{ }^{1}$ \\ ${ }^{1}$ Department of Cardiology, Handan First Hospital, Handan 056002, China \\ ${ }^{2}$ Department of Geriatrics, Handan Central Hospital, Handan 056002, China \\ ${ }^{3}$ Department of Cardiology, The People's Hospital of Langfang City, Langfang 065000, China \\ ${ }^{4}$ Department of Cardiology, The People's Hospital of Guangxi Zhuang Autonomous Region, Nanning 530021, China
}

Correspondence should be addressed to Chao Chang; changchao9999@sina.com

Received 13 November 2018; Accepted 24 May 2019; Published 22 July 2019

Academic Editor: Daniela Novick

Copyright (c) 2019 Haiying Hu et al. This is an open access article distributed under the Creative Commons Attribution License, which permits unrestricted use, distribution, and reproduction in any medium, provided the original work is properly cited.

Background. Interleukin- (IL-) 18 is a proinflammatory cytokine related to cardiovascular diseases, including hypertension and atherosclerosis. This study is aimed at determining whether IL-18 is related to aortic dissection (AD) and identifying the underlying mechanisms. Methods. IL-18 expression in human aorta samples from $\mathrm{AD}(n=8)$ and non-AD (NAD, $n=7)$ patients was measured. In addition, the IL-18, IL-6, interferon- (IFN-) $\gamma$, and IL-18-binding protein (IL-18BP) concentrations in plasma samples collected from the NAD and AD patients were detected. The effects of IL-18 on macrophage differentiation and smooth muscle cell (SMC) apoptosis were investigated in vitro. Results. IL-18 expression was significantly increased in the aorta samples from the AD patients compared with those from the NAD patients, especially in the torn section. Aortic IL-18 was mainly derived from macrophages and also partly derived from CD4+ T lymphocytes and vascular SMCs. Plasma IL- 18 , IFN- $\gamma$, and IL-6 levels were significantly higher in the AD group than in the NAD group, and the IL-18 levels were positively correlated with the IFN- $\gamma$ and IL-6 levels. In addition, plasma IL-18BP and free IL-18 levels were also elevated in the AD group. Linear regression analysis showed that the IL-18 level was independently associated with the presence of AD. In addition, anti-mouse IL-18-neutralizing monoclonal antibodies (anti-IL-18 nAb) inhibited angiotensin II-induced M1 macrophage differentiation and SMC apoptosis in vitro. Conclusion. IL-18 may participate in AD by regulating macrophage differentiation and macrophageinduced SMC apoptosis.

\section{Introduction}

Given its features of acute onset, rapid development, and high morbidity and mortality, acute aortic dissection (AD) is a severe cardiovascular disease. A previous study reported that the hourly mortality rate of early acute $\mathrm{AD}$ is approximately $1 \%-2 \%$ after the onset of symptoms [1]. However, common diagnostic methods, such as electrocardiography, chest radiography, and computed tomography (CT), are either unavailable at the bedside or time-consuming, and the misdiagnosis rate may be approximately 38\% [2]. Although the specific mechanism of $\mathrm{AD}$ remains unknown, studies have demonstrated that the inflammatory response is associated with $\mathrm{AD}[3,4]$.
Interleukin- (IL-) 18 belongs to the IL-1 family and was originally identified as an interferon- (IFN-) $\gamma$-inducing factor [5]. The main sources of IL-18 in humans are immune cells, such as monocytes, macrophages, dendritic cells, and B lymphocytes [6]. IL-18 has been reported to be involved in many inflammatory and immune diseases by promoting the expression of other proinflammatory cytokines. IL-18 can promote IFN- $\gamma$ secretion, which is one of the most important mechanisms of type 1 diabetes (T1D) [7]. In addition, IL-18 expression is also increased in human atopic eczema, possibly via the upregulation of the $\mathrm{T}$ helper 1 (Th1) immune response and downregulation of the T helper 2 (Th2) immune response, which aggravates the imbalance between Th1 and Th2 cells and results in the deterioration 
etiology of atopic eczema seen in humans [8, 9]. However, the biological actions of IL-18 can be countered by its endogenous antagonist, termed IL-18-binding protein (IL-18BP) [10].

IL-18 has also been reported to be closely related to a variety of cardiovascular diseases. An earlier study reported that IL-18 levels are a strong independent factor of death from cardiovascular causes in patients with coronary artery disease [11]. In a recent study, a change in IL-18 levels was found to be useful in the diagnosis of acute coronary syndrome (ACS) and prediction of its prognosis [12]. In addition, circulating IL-18 levels are elevated in patients with atrial fibrillation and may be superior to other inflammatory markers that are known to have elevated levels [13]. However, the IL-18 expression pattern in $\mathrm{AD}$ remains unknown, and this study is aimed at detecting IL-18 expression in human $\mathrm{AD}$ and exploring possible mechanisms underlying the participation of IL-18 in AD.

\section{Materials and Methods}

2.1. Collection of Human Aortic Samples and Blood Samples. A total of 8 aortic samples were obtained from patients who suffered from thoracic aortic dissection (TAD) and received aortic replacement surgery; 7 control aortic samples were collected from organ donors who suffered accidental death and did not exhibit obvious cardiovascular disease. From January 2017 to May 2018, consecutive patients $(n=150)$ who were hospitalized and received computed tomography angiography (CTA) after experiencing sudden chest pain were included in this study. Patients who had a history of autoimmune disease (including rheumatoid arthritis, $n=2$; psoriasis, $n=1$; and systemic lupus erythematosus, $n=3$ ) or cardiovascular disease (including coronary artery disease, $n=11$; valvular heart disease, $n=5$; viral myocarditis, $n=3$; pulmonary arterial hypertension (PAH), $n=2$; and heart failure, $n=4)$ were excluded. The remaining 119 patients were divided into a non-AD group (NAD group, $n=31)$ and an AD group $(n=88)$ according to their clinical symptoms and CTA results. The AD group was further divided into a Stanford A group (45 patients) and Stanford B group (43 patients).

All aortic samples and blood samples were collected at the People's Hospital of Guangxi Zhuang Autonomous Region as described in our previous studies [14, 15]. This study was approved by the Medical Ethics Committee of the People's Hospital of Guangxi Zhuang Autonomous Region and the Handan First Hospital, and the patients or their families provided informed consent.

2.2. Western Blot Analysis. Total protein was successfully extracted from aortic tissue samples in RIPA lysis buffer. Protein concentrations were detected and quantified using the BCA Protein Assay Kit (Thermo Fisher Scientific, USA). A total of $20 \mu \mathrm{g}$ of total protein was separated by sodium dodecyl sulfate-polyacrylamide gel electrophoresis. The proteins were transferred to a polyvinylidene fluoride membrane. The membranes were blocked with 5\% nonfat milk and then incubated with an anti-IL-18 antibody (Abcam, UK) and
anti-GAPDH antibody (Cell Signaling Technology, USA) overnight at $4^{\circ} \mathrm{C}$, followed by incubation with a secondary antibody at room temperature for 1 hour. The blots were detected using a two-color infrared imaging system (Odyssey, LICOR) to quantify protein expression.

2.3. Real-Time Quantitative PCR ( $q R T-P C R)$. Total RNA was extracted from thoracic aortic tissue samples and cells using TRIzol reagent, and cDNA was synthesized from $2 \mu \mathrm{g}$ of total mRNA using oligo (dT) primers and a reverse transcription kit according to the manufacturer's instructions. PCR amplifications were performed using LightCycler 480 SYBR Green Master Mix (all from Roche). The sequences of the primers are listed in Table 1. Relative mRNA expression levels were measured and normalized to the GAPDH expression level in the corresponding sample.

2.4. Immunofluorescence. Aortic samples were fixed with $4 \%$ neutral paraformaldehyde, embedded in paraffin, cut into 4 to $5 \mathrm{~mm}$ sections, and mounted onto slides. Immunofluorescence staining was used to detect the expression of IL-18 in each sample. In addition, double immunofluorescence staining using an anti-CD4 antibody and anti-IL-18 antibody, anti-CD68 antibody and anti-IL-18 antibody, or anti- $\alpha$ SMA antibody and anti-IL-18 antibody was performed.

2.5. Measurement of Cytokine Levels. All samples were thawed at $4^{\circ} \mathrm{C}$, and plasma IL-18, IFN- $\gamma$, IL-6, and IL-18BP (triple from eBioscience) levels were measured using commercially available enzyme-linked immune sorbent assay (ELISA) kits according to the manufacturer's instructions. The amount of free IL-18 was calculated based on the mass action law using a dissociation constant of $400 \mathrm{pM}$ and a stoichiometric ratio of $1: 1$ [16].

2.6. Isolation of Macrophages and CD4+ T Lymphocytes. Male wild-type (WT) mice (HFK Bioscience, Beijing, China) on the C57BL/ 6 background and aged 10-12 weeks were used in this study. Bone marrow-derived macrophages (Møs) were prepared as described previously $[17,18]$. The isolated macrophages were plated in complete DMEM supplemented with murine macrophage colony-stimulating factor $(50 \mathrm{ng} / \mathrm{ml})$ and cultured to allow macrophage differentiation. Spleens isolated from WT mice were mashed, and the splenocyte suspension was purified using a CD4+ cell isolation kit (Miltenyi Biotec, Auburn, CA). Then, the isolated T lymphocytes (TCs) were cultivated in complete RPMI 1640 medium and activated by treatment with anti-CD3 and anti-CD28 antibodies.

2.7. Cell Culture Experiments. Macrophages and CD4+ TCs isolated as described above were divided into the following groups: (1) macrophages, (2) macrophages+Ang II (100 nmol/l, Sigma), (3) macrophages+CD4+ TCs+AngII, and (4) macrophages+CD4+ TCs+Ang II+anti-mouse IL18-neutralizing monoclonal antibody (anti-IL-18 nAb; $100 \mathrm{ng} / \mathrm{ml}, \mathrm{R} \& \mathrm{D}$ Systems). The numbers of macrophages and CD4+ TCs in each group were $5 \times 10^{5}$ and $2.5 \times 10^{6}$, respectively. After treatment for 24 hours in complete DMEM, the iNOS, IFN- $\gamma$, and IL-6 mRNA levels in the 
TABLE 1: RT-qPCR primers used.

\begin{tabular}{lcc}
\hline Gene & Forward primer $\left(5^{\prime}-3^{\prime}\right)$ & Reverse primer $\left(5^{\prime}-3^{\prime}\right)$ \\
\hline IL-18 (human) & AAAGATAGCCAGCCTAGAGGTATG & GATCTATCCCCCAATCATCCT \\
GAPDH (human) & TTGTCAAGCTCATTTCCTGGT & TTACTCCTTGGAGGCCATGTA \\
iNOS (mouse) & TGACGCTCGGAACTGTAGCA & CAGTGATGGCCGACCTGAT \\
IFN- $\gamma$ (mouse) & ACTGGCAAAAGGATGGTGAC & TGAGCTCATTGAATGCTTGG \\
IL-6 (mouse) & AGTTGCCTTCTTGGGACTGA & TCCACGATTTCCCAGAGAAC \\
Bax (mouse) & TGAGCGAGTGTCTCCGGCGAAT & GCACTTTAGTGCACAGGGCCTTG \\
Bcl2 (mouse) & TGGTGGACAACATCGCCCTGTG & GGTCGCATGCTGGGGCCATATA \\
GAPDH (mouse) & AACTTTGGCATTGTGGAAGG & CACATTGGGGGTAGGAACAC \\
\hline
\end{tabular}

IL: interleukin; iNOS: inducible NO synthase; IFN: interferon; GAPDH: glyceraldehyde 3-phosphate dehydrogenase.

macrophages were detected by qRT-PCR. In addition, SMCs were treated with the supernatant of the cultures described above and Ang II for 12 hours, and the Bax and Bcl2 mRNA levels in the SMCs were measured.

2.8. Statistical Analysis. We first analyzed whether the cytokine and clinical characteristic data conformed to a normal distribution. Data with a normal distribution were expressed as the mean \pm standard deviation (SD), and differences between two groups were assessed using Student's $t$-tests, while differences between more than two groups were compared by one-way analysis of variance (ANOVA) followed by Tukey's multiple comparison test. Data with a nonnormal distribution were expressed as the median (lower quartile to upper quartile) and were compared by the MannWhitney $U$ test. Categorical variables are presented as counts (percentages) and were compared with the chi-square test. Spearman's correlation analysis was used to calculate correlations among plasma IL-18, IFN- $\gamma$, IL-6, and clinical characteristics. To identify independent predictors of the onset of $\mathrm{AD}$, univariate and multivariate linear regression analyses were performed. A value of $P<0.05$ was considered to indicate statistical significance. Statistical analysis was performed using GraphPad Prism7.

\section{Results}

3.1. Baseline Clinical Characteristics of the Patients Who Provided Aortic Tissue Samples. There were no significant differences in age, gender, uncontrolled blood pressure (HBP), smoking, the fasting glucose (Glu) level, systolic blood pressure (SBP), diastolic blood pressure (DBP), the total cholesterol (TC) level, the total triglyceride (TG) level, the high-density lipoprotein cholesterol (HDL-C) level, the low-density lipoprotein cholesterol (LDL-C) level, the creatinine (CREA) level, or heart rate (HR) between the TAD group and the control group. In contrast, the $\mathrm{C}$-reaction protein (CRP) level, white blood cell (WBC) count, and D-dimer level were significantly increased in the TAD group compared with the control group. The clinical characteristics of these patients are shown in Table 2.

3.2. Expression and Source of IL-18 in Human Thoracic Aortic Tissue. IL-18 expression in human $\mathrm{AD}$ aortic samples was first detected by Western blot analysis and RT-qPCR. The
TABLE 2: Summary of clinical characteristics in patients who provided aortic tissue samples.

\begin{tabular}{lccc}
\hline Characteristic & NAD & TAD & $P$ value \\
\hline Gender $(\mathrm{M} / \mathrm{F})$ & $5 / 2$ & $6 / 2$ & 0.876 \\
Age $($ years $)$ & $45(37,61)$ & $53(32,65)$ & 0.315 \\
HBP $(n, \%)$ & $3(42.8)$ & $6(75)$ & 0.221 \\
Smoking $(n, \%)$ & $3(42.8)$ & $5(62.5)$ & 0.462 \\
Glu $(\mathrm{mmol} / \mathrm{l})$ & $5.15(4.83,5.83)$ & $5.12(4.83,5.56)$ & 0.792 \\
SBP $(\mathrm{mmHg})$ & $137(125,162)$ & $155(139,164)$ & 0.254 \\
DBP $(\mathrm{mmHg})$ & $90(78,111)$ & $82(78,96)$ & 0.352 \\
TC $(\mathrm{mmol} / \mathrm{l})$ & $4.31(3.95,4.94)$ & $3.92(3.74,4.29)$ & 0.104 \\
TG $(\mathrm{mmol} / \mathrm{l})$ & $1.21(0.94,1.68)$ & $0.92(0.80,1.12)$ & 0.102 \\
HDL-C $(\mathrm{mmol} / \mathrm{l})$ & $1.88(1.19,2.14)$ & $1.60(1.10,1.92)$ & 0.363 \\
LDL-C $(\mathrm{mmol} / \mathrm{l})$ & $1.81(1.52,3.07)$ & $2.27(1.28,2.84)$ & 0.909 \\
CRP $(\mathrm{mg} / \mathrm{l})$ & $0.95(0.25,2.04)$ & $6.06(4.63,8.96)$ & 0.021 \\
WBC $\left(\times 10^{9} / \mathrm{l}\right)$ & $5.91(4.46,7.86)$ & $14.0(9.55,15.15)$ & 0.002 \\
CREA $(\mu \mathrm{mol} / \mathrm{l})$ & $68(58,74)$ & $74(59,97)$ & 0.314 \\
HR $(\mathrm{bpm})$ & $59(57,78)$ & $78(70,85)$ & 0.071 \\
D-dimer $(\mu \mathrm{g} / \mathrm{ml})$ & $0.86(0.69,0.96)$ & $6.95(3.26,9.75)$ & 0.011 \\
\hline
\end{tabular}

HBP: uncontrolled or failure to control of blood pressure; Glu: fasting glucose; SBP: systolic blood pressure; DBP: diastolic blood pressure; TC: total cholesterol; TG: total triglycerides; HDL-C: high-density lipoprotein cholesterol; LDL-C: low-density lipoprotein cholesterol; CRP: C-reactive protein; WBC: white blood cell; CREA: creatinine; HR: heart rate.

results showed that aortic IL-18 levels were significantly increased in the TAD patients compared with the control group (Figures 1(a) and 1(b)). In addition, the results of immunofluorescence staining showed that higher IL-18 levels were observed in the samples of aorta, especially in the torn region, from the TAD patients (Figure 1(c)). In addition, double staining was performed to detect the main source of IL-18. The results showed that IL-18 was mainly expressed in macrophages, although small amounts were also expressed in T lymphocytes and vascular smooth muscle cells (SMCs) in human thoracic aortic tissue (Figure 1(d)).

3.3. Basic Clinical Characteristics of the Patients Who Provided Blood Samples. There were no significant differences in age, HBP, SBP, DBP, HR, medications, or the levels of TC, TG, HDL-C, LDL-C, or CRP between the NAD group and $\mathrm{AD}$ group. The incidence rate of smoking and the 


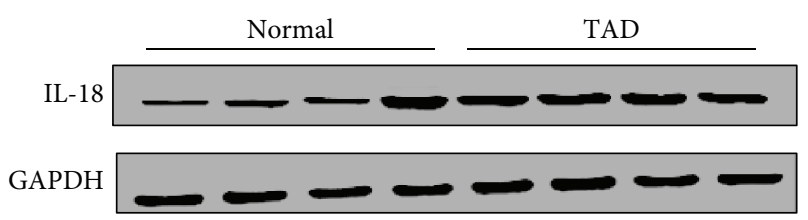

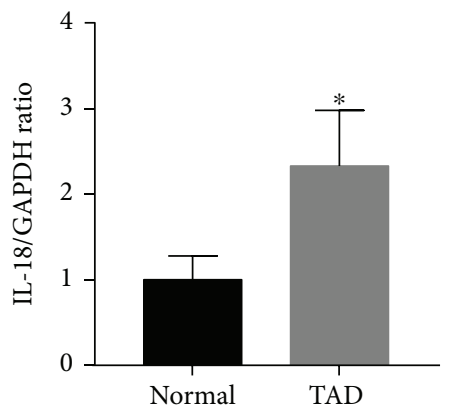

(a)

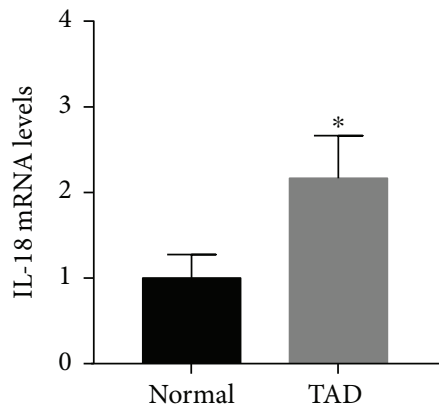

(b)

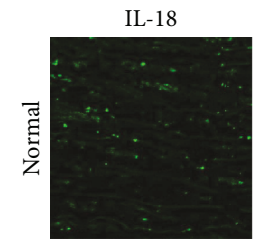

DAPI
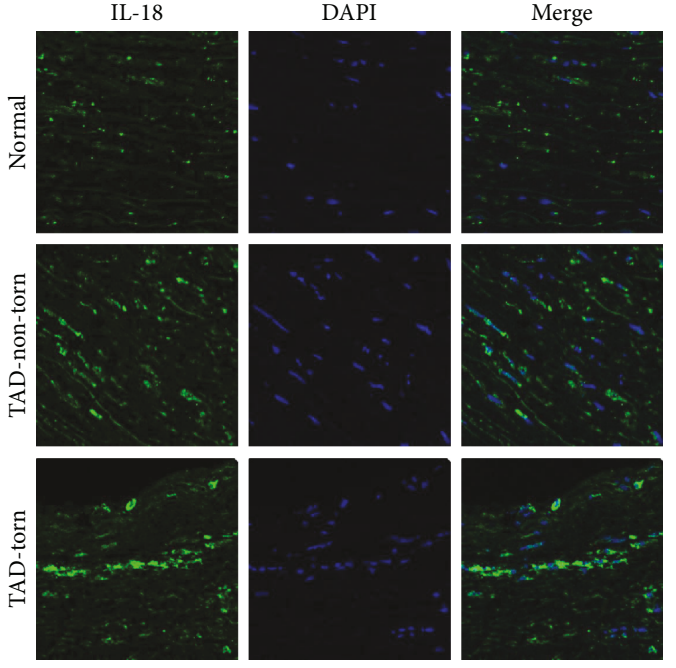

(c)
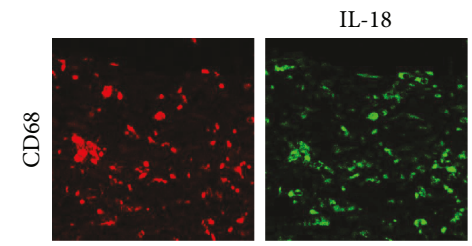

DAPI
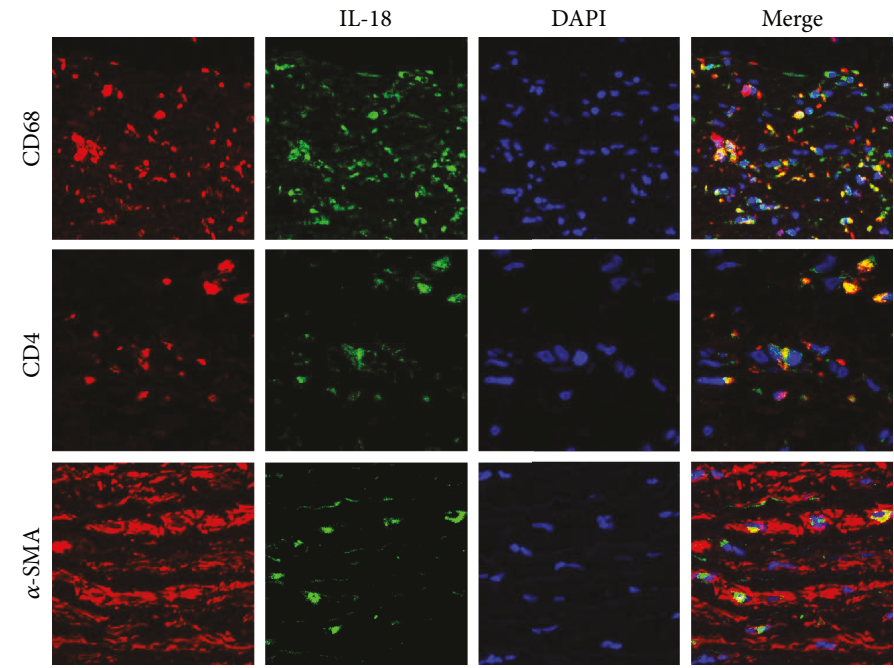

(d)

Figure 1: The IL-18 expression in human thoracic aortic tissues. (a) Aortic IL-18 protein levels in normal and TAD aorta tissues were detected by Western blot. (b) Aortic IL-18 mRNA levels in these two groups were measured by RT-PCR. (c) Immunofluorescence analysis of IL-18 expression in human thoracic aortic tissues (200x). (d) Double immunofluorescence staining analysis of IL-18 source in TAD aortas $(400 \mathrm{x}) .{ }^{*} P<0.05$ vs. the normal group. 
TABLE 3: Summary of clinical characteristics in patients who provided blood sample.

\begin{tabular}{|c|c|c|c|c|}
\hline Characteristic & NAD & $\mathrm{AAD}$ & Stanford A & Stanford B \\
\hline Gender $(\mathrm{M} / \mathrm{F})$ & $19 / 12$ & $70 / 18^{*}$ & $37 / 8^{*}$ & $33 / 10$ \\
\hline Age (years) & $64(50,76)$ & $56(50,64)$ & $55(46,61)^{*}$ & $61(53,69)$ \\
\hline Smoking $(n, \%)$ & $6(19.3)$ & $36(40.9)^{*}$ & $18(40)$ & $18(41.8)^{*}$ \\
\hline $\operatorname{HBP}(n, \%)$ & $25(80.6)$ & $79(89.7)$ & $41(91.1)$ & $38(88.3)$ \\
\hline Glu (mmol/l) & $6.13(5.6,6.8)$ & $7.2(6.4,8.3)^{*}$ & $7.3(6.4,8.2)^{*}$ & $6.8(6.3,8.7)^{*}$ \\
\hline $\mathrm{SBP}(\mathrm{mmHg})$ & $156(146,170)$ & $145(128,167)$ & $144(121,161)^{*}$ & $145(132,170)$ \\
\hline DBP (mmHg) & $84(76,93)$ & $80(70,94)$ & $75(66,92)$ & $80(74,97)$ \\
\hline WBC $\left(\times 10^{9} / \mathrm{L}\right)$ & $7.5(4.8,11.6)$ & $11.0(8.1,13.5)^{*}$ & $11.6(9.5,14.1)^{*}$ & $10.0(6.7,12.9)$ \\
\hline $\mathrm{TC}(\mathrm{mmol} / \mathrm{l})$ & $4.0(3.5,4.3)$ & $4.07(3.4,4.7)$ & $4.13(3.6,4.7)$ & $4.0(3.4,4.6)$ \\
\hline TG (mmol/l) & $1.4(1.2,1.6)$ & $1.2(0.9,1.7)$ & $1.2(0.9,1.9)$ & $1.1(0.9,1.7)$ \\
\hline HDL-C $(\mathrm{mmol} / \mathrm{l})$ & $1.0(0.9,1.1)$ & $1.1(0.9,1.4)$ & $1.0(0.8,1.3)$ & $1.3(0.9,1.5)$ \\
\hline LDL-C (mmol/l) & $2.1(1.9,2.3)$ & $2.2(1.7,2.7)$ & $2.3(1.8,2.8)$ & $2.0(1.6,2.6)$ \\
\hline HR (bmp) & $72(63,84)$ & $78(68,90)$ & $78(60,84)$ & $80(72,91)^{*}$ \\
\hline CREA $(\mu \mathrm{mol} / \mathrm{l})$ & $72(62,92)$ & $82(68,106)$ & $80(67,94)$ & $85(75,113)^{*}$ \\
\hline $\mathrm{D}$-dimer $(\mu \mathrm{g} / \mathrm{ml})$ & $0.8(0.6,1.0)$ & $4.2(2.0,6.3)^{*}$ & $4.7(2.4,7.2)^{*}$ & $4.0(1.6,5.5)^{*}$ \\
\hline CRP (mg/l) & $18.4(3.8,60.9)$ & $10.7(2.7,57.4)$ & $11.8(2.6,61.3)$ & $9.1(2.8,58.8)$ \\
\hline \multicolumn{5}{|l|}{ Medications, $n(\%)$} \\
\hline Aspirin & $5(16.1)$ & $13(14.7)$ & $5(11.1)$ & $8(18.6)$ \\
\hline ACEI/ARB & $9(29.0)$ & $35(39.7)$ & $14(31.1)$ & $21(48.8)$ \\
\hline Beta-blockers & $4(12.9)$ & $18(20.4)$ & $7(15.5)$ & $11(25.5)$ \\
\hline $\mathrm{CCB}$ & $9(29.0)$ & $33(37.5)$ & $18(40.0)$ & $15(34.8)$ \\
\hline Diuretics & $3(9.6)$ & $15(17.0)$ & $6(13.3)$ & $9(20.9)$ \\
\hline
\end{tabular}

ACEI: angiotensin-converting enzyme inhibitor; ARB: angiotensin receptor blocker; CCB: calcium channel blocker. ${ }^{*} P<0.05$ vs. NAD group.

proportion of males as well as the Glu level, WBC count, and $\mathrm{D}$-dimer level were significantly increased in the $\mathrm{AD}$ group compared with the NAD group. However, there were no differences between the Stanford A group and the Stanford B group in the clinical characteristics. The clinical characteristics of each group are listed in Table 3.

3.4. Plasma Cytokine Concentrations in the AD Patients. The plasma levels of IL-18, IFN- $\gamma$, and IL- 6 were detected. The results showed that compared with the NAD group, the AD group had higher IL-18, IFN- $\gamma$, and IL-6 levels (Figures 2(a)-2(c)), whereas no significant differences in these three cytokines were found between the Stanford A group and the Stanford B group (Figures 2(a)-2(c)). The plasma levels in each group are shown in Table 4. Furthermore, the plasma IL-18 levels were positively correlated with the IFN- $\gamma$ levels $(r=0.4761, P<0.001)$ and the IL-6 levels $(r=0.2985, P<0.01)$ in the AD patients (Figures 2(d) and 2(e)). The IFN- $\gamma$ levels were also positively correlated with the IL-6 levels $(r=0.3621, P<0.01)$ (Figure 2(f)). In addition, we detected the IL-18BP levels in the plasma. The results are listed in Table 4 . The results showed that the IL-18BP level and calculated free IL-18 level were also elevated in the AD group compared with the NAD group (Figures 2(g) and 2(h)).

3.5. Univariate and Multivariate Linear Regression Analyses. To determine whether IL-18 is an independent predictor of the presence of $\mathrm{AD}$, univariate and multivariate linear regression analyses were performed. The univariate analysis showed that the IL-18, IFN- $\gamma$, IL-6, Glu, CREA, and Ddimer levels; gender; and smoking status exhibited a trend towards an association with the presence of $\mathrm{AD}$, whereas SBP, DBP, the TG level, the TC level, the CRP level, the WBC count, age, and HR showed no obvious association. Furthermore, the IL-18, IFN- $\gamma$, IL-6, Glu, CREA, and Ddimer levels; gender; and smoking status were used to perform multivariate linear regression analysis, and the results showed that the IL-18 level was independently associated with the presence of $\operatorname{AD}(\beta=0.238,95 \%$ confidence interval (CI) 0.053 to $0.422 ; P=0.012$ ). In addition, the IFN- $\gamma$ $(\beta=0.232,95 \%$ CI 0.044 to $0.419 ; P=0.016)$, IL-6 ( $\beta=0.163,95 \%$ CI 0.002 to $0.324 ; P=0.048)$, and D-dimer ( $\beta=0.179,95 \%$ CI 0.014 to $0.343 ; P=0.034$ ) levels were also associated with the presence of $\mathrm{AD}$ (as shown in Table 5).

3.6. Effect of IL-18 on Macrophage Differentiation and SMC Apoptosis. The results of cell culture experiments showed that Ang II treatment alone did not affect iNOS expression, but the iNOS level exhibited a significant increase after CD4+ T lymphocytes were added, and the iNOS level increase was partly reversed by the anti-IL-18 nAb (Figure 3(a)). A similar trend was observed for the IFN- $\gamma$ and IL- 6 mRNA levels (Figure 3(a)). In addition, the medium from the macrophage group and macrophage+Ang II group did not affect Bax and $\mathrm{Bcl} 2$ mRNA expression in SMCs; the Bax mRNA levels were 


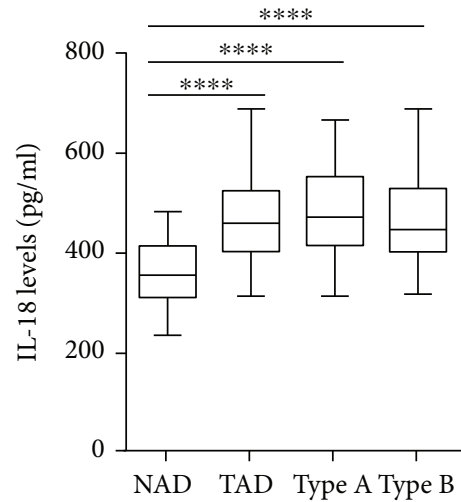

(a)

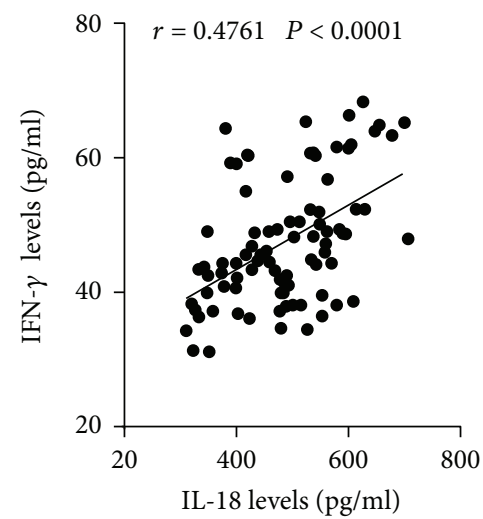

(d)

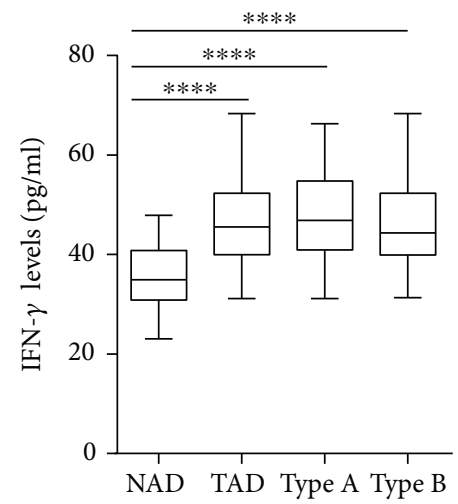

(b)

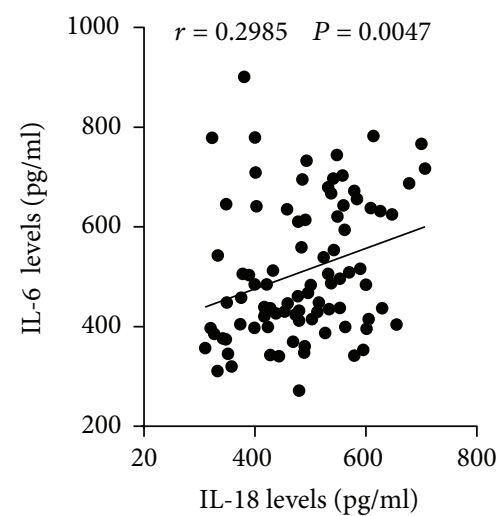

(e)

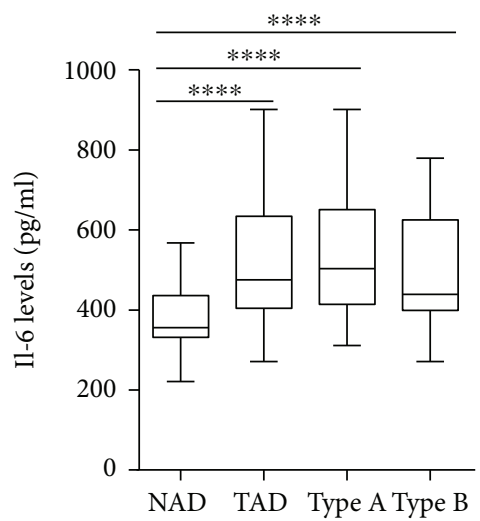

(c)

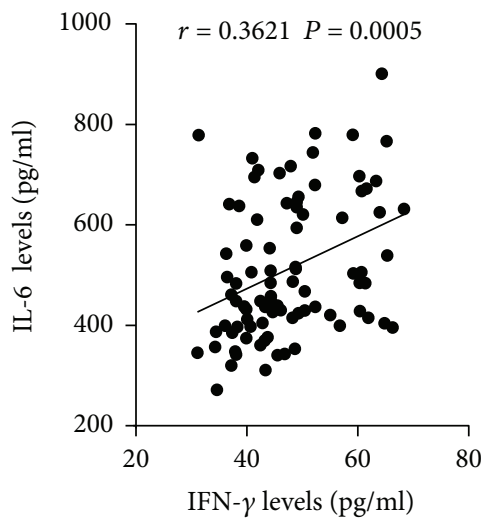

(f)

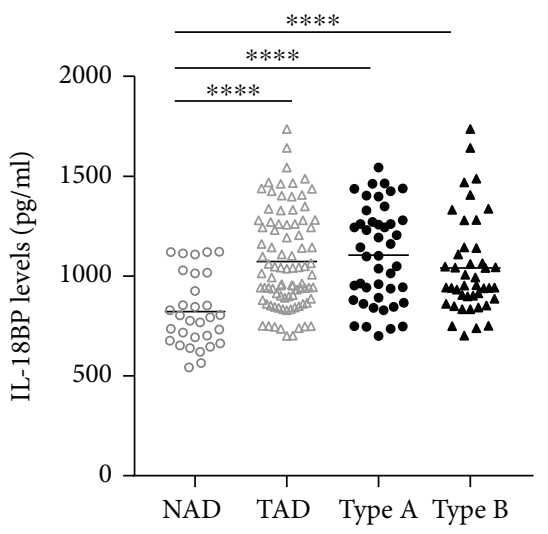

(g)

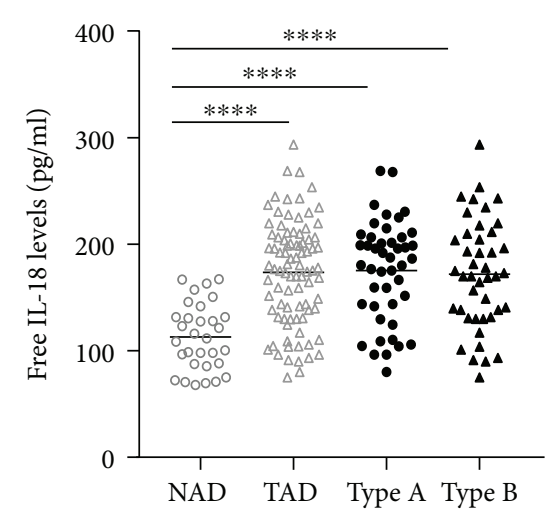

(h)

FIgURE 2: Plasma cytokine levels in NAD and AAD groups. Plasma IL-18 (a), IFN- $\gamma$ (b), and IL-6 (c) concentrations in NAD, AAD, Stanford A, and Stanford B groups. The correlation between IL-18 (d), IL-6 (e), and IFN- $\gamma$ levels (f) in AAD patients. Plasma IL-18BP levels (g) and the free IL-18 (h) in NAD, AAD, Stanford A, and Stanford B groups. ${ }^{* * * *} P<0.0001$ vs. the NAD group.

TABLe 4: Plasma cytokines in control and AAD groups.

\begin{tabular}{lccrr}
\hline Cytokines & NAD & AAD & Type A & Type B \\
\hline IL-18 $(\mathrm{pg} / \mathrm{ml})$ & $354.1(293.7,429.3)$ & $487.3(406.5,556.9)^{*}$ & $512.8(425.6,560.1)^{*}$ & $479.8(401.4,548.8)^{*}$ \\
IL-18BP $(\mathrm{pg} / \mathrm{ml})$ & $821.9(676.4,1014.3)$ & $1073(887,1260)^{*}$ & $1104(886,1275)^{*}$ & $1040(886,1140)^{*}$ \\
IFN- $\gamma(\mathrm{pg} / \mathrm{ml})$ & $34.9(30.8,40.8)$ & $45.5(39.9,52.3)^{*}$ & $46.8(40.9,54.7)^{*}$ & $44.3(39.9,52.3)^{*}$ \\
IL-6 $(\mathrm{pg} / \mathrm{ml})$ & $355.9(331.6,436.1)$ & $475.7(404.4,634.3)$ & $503.2(414.3,650.9)^{*}$ & $439.3(399.3,625.2)^{*}$ \\
\hline
\end{tabular}

${ }^{*} P<0.05$ vs. NAD group. 
TABLE 5: Association between cytokines, clinical characteristics, and the presence of AAD was assessed by univariate analysis and multivariate linear regression analysis.

\begin{tabular}{lccccc}
\hline Variables & Beta & $\begin{array}{c}\text { Simple linear } \\
95 \% \text { CI }\end{array}$ & $P$ value & Beta & $\begin{array}{c}\text { Multiple linear } \\
95 \% \text { CI }\end{array}$ \\
\hline IL-18 & 0.509 & 0.351 to 0.667 & 0.001 & 0.238 & 0.053 to 0.422 \\
IFN- $\gamma$ & 0.522 & 0.366 to 0.678 & 0.001 & 0.232 & 0.044 to 0.419 \\
IL-6 & 0.419 & 0.252 to 0.585 & 0.001 & 0.163 & 0.002 to 0.324 \\
Gender & 0.185 & 0.005 to 0.364 & 0.045 & 0.124 & -0.029 to 0.276 \\
Smoking & 0.198 & 0.018 to 0.377 & 0.031 & 0.137 & -0.014 to 0.288 \\
Glu & 0.345 & 0.173 to 0.517 & 0.001 & 0.045 & -0.116 to 0.205 \\
CREA & 0.216 & 0.037 to 0.395 & 0.018 & -0.024 & -0.176 to 0.128 \\
D-dimer & 0.464 & 0.302 to 0.626 & 0.001 & 0.179 & 0.014 to 0.343 \\
SBP & -0.149 & -0.330 to 0.032 & 0.107 & & 0.111 \\
DBP & -0.102 & -0.284 to 0.080 & 0.269 & & 0.583 \\
TG & -0.068 & -0.250 to 0.115 & 0.465 & & 0.754 \\
TC & 0.034 & -0.149 to 0.217 & 0.716 & & 0.034 \\
CRP & -0.028 & -0.211 to 0.155 & 0.760 & & \\
WBC & 0.140 & -0.041 to 0.322 & 0.128 & & \\
Age & -0.130 & -0.312 to 0.052 & 0.159 & & \\
HR & 0.170 & -0.011 to 0.350 & 0.065 & & \\
\hline
\end{tabular}

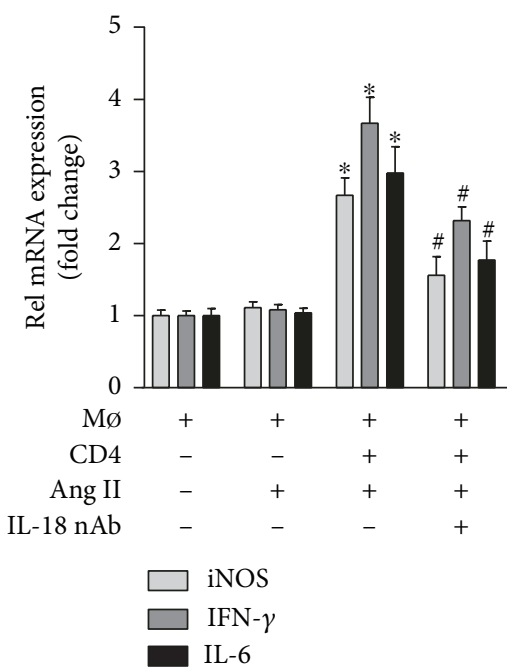

(a)

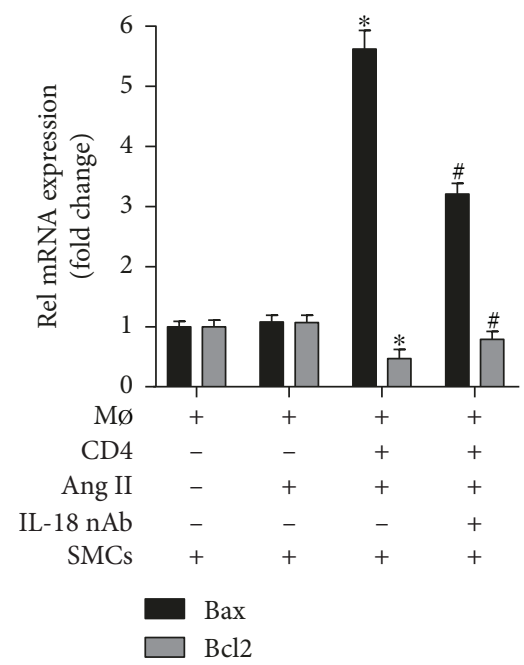

(b)

FIGURE 3: Effect of IL-18 nAb on macrophage differentiation and SMC apoptosis. (a) iNOS, IFN- $\gamma$, and IL-6 mRNA levels in macrophage were measured by RT-qPCR. ${ }^{*} P<0.05$ vs. the Mø group; ${ }^{\#} P<0.05$ vs. the M $\varnothing+\mathrm{CD} 4+\mathrm{TCs}+$ Ang II group. (b) The Bax and Bcl 2 mRNA levels in SMCs were measured by RT-qPCR. ${ }^{*} P<0.05$ vs. the M $\varnothing+S M C$ group; ${ }^{*} P<0.05$ vs. the M $\varnothing+C D 4+$ TCs+Ang II+SMC group.

increased, while the Bcl 2 mRNA levels were decreased after treatment with the medium from the macrophage+Ang II $+\mathrm{CD} 4+\mathrm{T}$ lymphocyte group, and these effects on Bax and $\mathrm{Bcl} 2$ expression were also reversed by the anti-IL-18 nAb (Figure 3(b)).

\section{Discussion}

In this study, we found that the level of the proinflammatory cytokine IL-18 was significantly increased in AD aortic tissue and demonstrated that IL-18 is produced mainly by infiltrated macrophages and partly by $\mathrm{T}$ lymphocytes and vascular SMCs. In addition, plasma IL-18 levels were dramatically increased in $\mathrm{AD}$ blood samples. The plasma IL-18 levels were also positively correlated with the levels of the M1 macrophage-associated cytokines IFN- $\gamma$ and IL-6. Furthermore, the results of a multivariate linear regression analysis showed that IL-18 may be an independent risk factor for diagnosing AD. An anti-IL-18 nAb inhibited M1 macrophage differentiation and SMC apoptosis.

Like most of the other members of the IL-1 superfamily, IL-18 is a powerful proinflammatory cytokine that can participate in many diseases [19]. In an earlier study, Harms et al. reported that circulating IL-18 levels are significantly 
increased in juvenile T1D patients compared to control subjects and that IL-18 expression is also elevated within pancreatic samples in T1D [20]. A prospective study and an updated meta-analysis found that circulating IL-18 levels are positively associated with coronary heart disease incidence [21]. In addition, circulating and lung IL-18 levels are elevated in PAH patients [22]. Furthermore, an animal experiment found that IL-18 disruption can suppress hypoxia-induced PAH [23]. IL-18 expression has also been reported to be increased approximately 2-fold in human atrial fibrillation patients compared with control subjects and closely related to the incidence of atrial fibrillation [13]. While the IL-18 expression pattern in human AD remains unknown, our present study found that IL-18 levels were increased in both the aorta and plasma of $\mathrm{AD}$ patients. These results may suggest that IL-18 is related to the onset of $\mathrm{AD}$.

Previous studies have demonstrated that the immune response and inflammation are closely related to the progression and development of AD. In an earlier study of IL-18, it was reported that granulocyte macrophage colonystimulating factor (GM-CSF) is a key regulatory and molecular causative agent of aortic dissection/intramural hematoma in a murine model of this condition and is also related to this condition in humans [24]. In addition, T lymphocytes can cause cell death and have been demonstrated to be one of the most important mechanisms underlying the onset of $\mathrm{AD}$ [25]. To date, much evidence has suggested that IL18 can be expressed in dendritic cells, monocytes, and Kupffer cells but especially in macrophages and $\mathrm{T}$ lymphocytes $[5,26,27]$. SMCs are the main cell type in the aorta; therefore, double immunofluorescence staining with an anti-IL-18 antibody and an anti-CD68 antibody, anti- $\alpha$ SMA antibody, or anti-CD4 antibody was conducted. We observed that aortic IL-18 expression was predominantly colocalized with macrophages and partially colocalized with T lymphocytes and SMCs in the aorta of AD patients. These results suggested that IL-18 may participate in the occurrence of $\mathrm{AD}$ via macrophages.

Data from clinical experiments and animal studies have demonstrated that IL- 6 and IFN- $\gamma$ play critical roles in the development and progression of AD. In an earlier study, aortic IL- 6 and IFN- $\gamma$ expression was reported to be significantly increased in a mouse AD model [28]. In other studies, Pope et al. and Ju et al. found that high IL-6 levels promoted the progression of AD, while the deletion of IL-6 significantly decreased the onset of $\operatorname{AD}[29,30]$. Xu et al. reported that circulating IFN- $\gamma$ levels are increased in human AD patients [15]. They also found that the IFN- $\gamma$ level increases in AD patients and is positively correlated with the occurrence of $\mathrm{AD}$ [15]. Given that macrophages may be the most important source of IL- 6 and IFN- $\gamma$, to investigate the mechanisms underlying the involvement of IL-18 in AD, we measured circulating IL- 6 and IFN- $\gamma$ levels and found that both the IL- 6 and IFN- $\gamma$ levels in the plasma were increased and positively associated with the IL-18 level in AD patients. These results suggest that IL-18 may be involved in AD by promoting the secretion of IL- 6 and IFN- $\gamma$ by macrophages.

IL-18BP, which is an endogenous antagonist of IL-18, can be induced by IFN- $\gamma$ [31]. To gain a better insight into the biological activity of IL-18 during acute $\mathrm{AD}$, we detected the plasma levels of IL-18BP in the non-AD group and the $\mathrm{AD}$ group. We found that high plasma IL-18 levels concurred with increased IL-18BP levels in the AD group. In addition, the calculated levels of free IL-18 were also increased in the acute $\mathrm{AD}$ group. IL-18 plays a major role in the production of IFN- $\gamma$, and IFN- $\gamma$ can stimulate the production of IL18BP, while IL-18BP can neutralize IL-18 and attenuate the inflammatory response [32]. Therefore, we can presume that IL-18 can promote the production of IFN- $\gamma$ and IL$18 \mathrm{BP}$ and overcome endogenous inhibition to promote the occurrence of AD.

Vascular SMCs are an important component of the aortic structure and play a vital role in maintaining the normal structure and function of the aorta in both humans and mice [33]. In addition, to maintain vasoconstriction and diastolic function, SMCs can continuously synthesize and degrade the extracellular matrix (ECM) while maintaining a dynamic balance, which is closely related to the onset and progression of $\mathrm{AD}$ because excessive SMC apoptosis can decrease ECM component expression and aortic stability [34, 35]. In fact, an interesting phenomenon was found in the progression of $\mathrm{AD}$; $\mathrm{M} 1$ macrophage activity was significantly enhanced, while M2 macrophage activity showed compensatory enhancement but was significantly weaker than the M1 macrophage activity and was not sufficient to counteract the proinflammatory activity of the M1 macrophages [36, 37]. The studies described above demonstrated that in an inflammatory environment, macrophages can convert into proinflammatory M1 macrophages and anti-inflammatory M2 macrophages, which play pathogenic and protective effects, respectively, in the onset and progression of $\mathrm{AD}$, and M1 macrophages play a leading role in the progression of AD. To investigate the mechanisms by which IL-18 participates in the progression of $\mathrm{AD}$, macrophages were treated with Ang II, and the effect of an anti-IL-18 nAb on macrophage differentiation was measured. Because a previous study demonstrated that CD4+ T lymphocytes are essential in the differentiation of macrophages [18], CD4+ T lymphocytes were also added to our cell culture. The results showed that the anti-IL-18 nAb significantly decreased iNOS mRNA expression in macrophages, and similar results for IL-6 and IFN- $\gamma$ were observed after treatment with the anti-IL-18 $\mathrm{nAb}$. The results showed that IL-18 may promote Ang IIand CD4+ T lymphocyte-induced M1 macrophage differentiation, increase M1 macrophage-induced IL-6 and IFN- $\gamma$ expression, and be involved in AD. To further explore the related mechanisms, SMCs were treated with the culture supernatant and Ang II, and the results showed that the anti-IL-18 nAb alleviated M1 macrophage-induced SMC apoptosis. Our results may show that IL-18 promotes M1 macrophage differentiation, increases SMC apoptosis, and accelerates the onset of AD.

In conclusion, IL-18 is widely expressed in various tissues and many cell types and can participate in different immunoregulatory mechanisms. This study is the first to demonstrate a positive association between IL-18 levels and the onset of AD. The possible mechanism is that IL-18 promotes M1 macrophage differentiation and increases SMC apoptosis. 
Certainly, there are some limitations to our study. First, the small sample size may not have sufficient power to detect a significant relationship between IL-18 and AD. Second, the mechanism of $\mathrm{AD}$ is complicated and difficult to clarify, and we explored only the regulatory effect of IL-18 on inflammation. Third, a larger sample size and prospective clinical studies are needed to further verify that IL-18 can contribute to the prognostic assessment of AAD.

\section{Data Availability}

The data used to support the findings of this study are available from the corresponding author upon request.

\section{Conflicts of Interest}

The authors declare that they have no conflicts of interest.

\section{Authors' Contributions}

Haiying $\mathrm{Hu}$ and Guangtai Zhang contributed equally to this work.

\section{References}

[1] P. G. Hagan, C. A. Nienaber, E. M. Isselbacher et al., "The international registry of acute aortic dissection (IRAD): new insights into an old disease," JAMA, vol. 283, no. 7, pp. 897903, 2000.

[2] P. C. Spittell, J. A. Spittell Jr., J. W. Joyce et al., "Clinical features and differential diagnosis of aortic dissection: experience with 236 cases (1980 through 1990)," Mayo Clinic Proceedings, vol. 68, no. 7, pp. 642-651, 1993.

[3] E. M. Isselbacher, "Thoracic and abdominal aortic aneurysms," Circulation, vol. 111, no. 6, pp. 816-828, 2005.

[4] F. Luo, X. L. Zhou, J. J. Li, and R. T. Hui, "Inflammatory response is associated with aortic dissection," Ageing Research Reviews, vol. 8, no. 1, pp. 31-35, 2009.

[5] H. Okamura, H. Tsutsui, T. Komatsu et al., "Cloning of a new cytokine that induces IFN- $\gamma$ production by T cells," Nature, vol. 378, no. 6552, pp. 88-91, 1995.

[6] A. Janiak, B. Lesniowski, A. Jasinska, M. Pietruczuk, and E. Malecka-Panas, "Interleukin 18 as an early marker or prognostic factor in acute pancreatitis," Gastroenterology Review, vol. 10, no. 4, pp. 203-207, 2015.

[7] A. M. Marleau and N. E. Sarvetnick, "IL-18 is required for selfreactive T cell expansion in NOD mice," Journal of Autoimmunity, vol. 36, no. 3-4, pp. 263-277, 2011.

[8] T. Bieber, "Atopic dermatitis," The New England Journal of Medicine, vol. 358, no. 14, pp. 1483-1494, 2008.

[9] T. Tanaka, H. Tsutsui, T. Yoshimoto et al., "Interleukin-18 is elevated in the sera from patients with atopic dermatitis and from atopic dermatitis model mice, NC/Nga," International Archives of Allergy and Immunology, vol. 125, no. 3, pp. 236240, 2001.

[10] D. Novick, S. H. Kim, G. Fantuzzi, L. L. Reznikov, C. A. Dinarello, and M. Rubinstein, "Interleukin-18 binding protein: a novel modulator of the Th1 cytokine response," Immunity, vol. 10, no. 1, pp. 127-136, 1999.
[11] S. Blankenberg, L. Tiret, C. Bickel et al., "Interleukin-18 is a strong predictor of cardiovascular death in stable and unstable angina," Circulation, vol. 106, no. 1, pp. 24-30, 2002.

[12] H. Sun, J. Zhang, Y. Zheng, and S. Shang, "Expressions and clinical significance of factors related to acute coronary syndrome," Journal of Biological Regulators and Homeostatic Agents, vol. 32, pp. 299-305, 2018.

[13] Y. Luan, Y. Guo, S. Li et al., "Interleukin-18 among atrial fibrillation patients in the absence of structural heart disease," Europace, vol. 12, no. 12, pp. 1713-1718, 2010.

[14] J. Ye, M. Wang, H. Jiang et al., "Increased levels of interleukin22 in thoracic aorta and plasma from patients with acute thoracic aortic dissection," Clinica Chimica Acta, vol. 486, pp. 395-401, 2018.

[15] Y. Xu, J. Ye, M. Wang et al., "Increased interleukin-11 levels in thoracic aorta and plasma from patients with acute thoracic aortic dissection," Clinica Chimica Acta, vol. 481, pp. 193199, 2018.

[16] D. Novick, B. Schwartsburd, R. Pinkus et al., “A novel IL-18BP ELISA shows elevated serum IL-18BP in sepsis and extensive decrease of free IL-18," Cytokine, vol. 14, no. 6, pp. 334-342, 2001.

[17] F. R. Lake, P. W. Noble, P. M. Henson, and D. W. Riches, "Functional switching of macrophage responses to tumor necrosis factor-alpha (TNF alpha) by interferons. Implications for the pleiotropic activities of TNF alpha," The Journal of Clinical Investigation, vol. 93, no. 4, pp. 1661-1669, 1994.

[18] Y. Li, C. Zhang, Y. Wu et al., "Interleukin-12p35 deletion promotes CD4 T-cell-dependent macrophage differentiation and enhances angiotensin II-induced cardiac fibrosis," Arteriosclerosis, Thrombosis, and Vascular Biology, vol. 32, no. 7, pp. 1662-1674, 2012.

[19] S. K. Sedimbi, T. Hägglöf, and M. C. I. Karlsson, "IL-18 in inflammatory and autoimmune disease," Cellular and Molecular Life Sciences, vol. 70, no. 24, pp. 4795-4808, 2013.

[20] R. Z. Harms, D. N. Yarde, Z. Guinn et al., "Increased expression of IL-18 in the serum and islets of type 1 diabetics," Molecular Immunology, vol. 64, no. 2, pp. 306-312, 2015.

[21] S. Kaptoge, S. R. K. Seshasai, P. Gao et al., "Inflammatory cytokines and risk of coronary heart disease: new prospective study and updated meta-analysis," European Heart Journal, vol. 35, no. 9, pp. 578-589, 2014.

[22] D. J. Ross, R. M. Strieter, M. C. Fishbein, A. Ardehali, and J. A. Belperio, "Type I immune response cytokine-chemokine cascade is associated with pulmonary arterial hypertension," The Journal of Heart and Lung Transplantation, vol. 31, no. 8, pp. 865-873, 2012.

[23] D. Morisawa, S. Hirotani, M. Oboshi et al., "Interleukin-18 disruption suppresses hypoxia-induced pulmonary artery hypertension in mice," International Journal of Cardiology, vol. 202, pp. 522-524, 2016.

[24] B. K. Son, D. Sawaki, S. Tomida et al., "Granulocyte macrophage colony-stimulating factor is required for aortic dissection/intramural haematoma," Nature Communications, vol. 6, no. 1, article 6994, 2015.

[25] R. He, D. C. Guo, W. Sun et al., "Characterization of the inflammatory cells in ascending thoracic aortic aneurysms in patients with Marfan syndrome, familial thoracic aortic aneurysms, and sporadic aneurysms," The Journal of Thoracic and Cardiovascular Surgery, vol. 136, no. 4, pp. 922 929.e1, 2008. 
[26] S. Ushio, M. Namba, T. Okura et al., "Cloning of the cDNA for human IFN-gamma-inducing factor, expression in Escherichia coli, and studies on the biologic activities of the protein," Journal of Immunology, vol. 156, no. 11, pp. 4274-4279, 1996.

[27] S. Stoll, H. Jonuleit, E. Schmitt et al., "Production of functional IL-18 by different subtypes of murine and human dendritic cells (DC): DC-derived IL-18 enhances IL-12-dependent Th1 development," European Journal of Immunology, vol. 28, no. 10, pp. 3231-3239, 1998.

[28] Y. L. Cai and Z. W. Wang, "The expression and significance of IL-6, IFN- $\gamma$, SM22 $\alpha$, and MMP-2 in rat model of aortic dissection," European Review for Medical and Pharmacological Sciences, vol. 21, no. 3, pp. 560-568, 2017.

[29] N. H. Pope, M. Salmon, W. F. Johnston et al., "Interleukin-6 receptor inhibition prevents descending thoracic aortic aneurysm formation," The Annals of Thoracic Surgery, vol. 100, no. 5, pp. 1620-1626, 2015.

[30] X. Ju, T. Ijaz, H. Sun et al., "IL-6 regulates extracellular matrix remodeling associated with aortic dilation in a fibrillin-1 hypomorphic $\mathrm{mgR} / \mathrm{mgR}$ mouse model of severe Marfan syndrome," Journal of the American Heart Association, vol. 3, no. 1, article e000476, 2014.

[31] H. Muhl, H. Kampfer, M. Bosmann, S. Frank, H. Radeke, and J. Pfeilschifter, "Interferon-gamma mediates gene expression of IL-18 binding protein in nonleukocytic cells," Biochemical and Biophysical Research Communications, vol. 267, no. 3, pp. 960-963, 2000.

[32] H. Zhang, J. Wang, L. Wang, H. Xie, L. Chen, and S. He, "Role of IL-18 in atopic asthma is determined by balance of IL18/IL-18BP/IL-18R," Journal of Cellular and Molecular Medicine, vol. 22, no. 1, pp. 354-373, 2018.

[33] G. Wang, L. Jacquet, E. Karamariti, and Q. Xu, "Origin and differentiation of vascular smooth muscle cells," The Journal of Physiology, vol. 593, no. 14, pp. 3013-3030, 2015.

[34] L. Wang, J. Zhang, W. Fu, D. Guo, J. Jiang, and Y. Wang, "Association of smooth muscle cell phenotypes with extracellular matrix disorders in thoracic aortic dissection," Journal of Vascular Surgery, vol. 56, no. 6, pp. 1698-1709.e1, 2012.

[35] J. D. Humphrey, M. A. Schwartz, G. Tellides, and D. M. Milewicz, "Role of mechanotransduction in vascular biology: focus on thoracic aortic aneurysms and dissections," Circulation Research, vol. 116, no. 8, pp. 1448-1461, 2015.

[36] M. A. Dale, M. K. Ruhlman, and B. T. Baxter, "Inflammatory cell phenotypes in AAAs: their role and potential as targets for therapy," Arteriosclerosis, Thrombosis, and Vascular Biology, vol. 35, no. 8, pp. 1746-1755, 2015.

[37] B. C. Tieu, C. Lee, H. Sun et al., "An adventitial IL-6/MCP1 amplification loop accelerates macrophage-mediated vascular inflammation leading to aortic dissection in mice," Journal of Clinical Investigation, vol. 119, no. 12, pp. 3637-3651, 2009. 


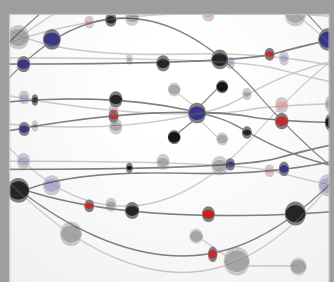

The Scientific World Journal
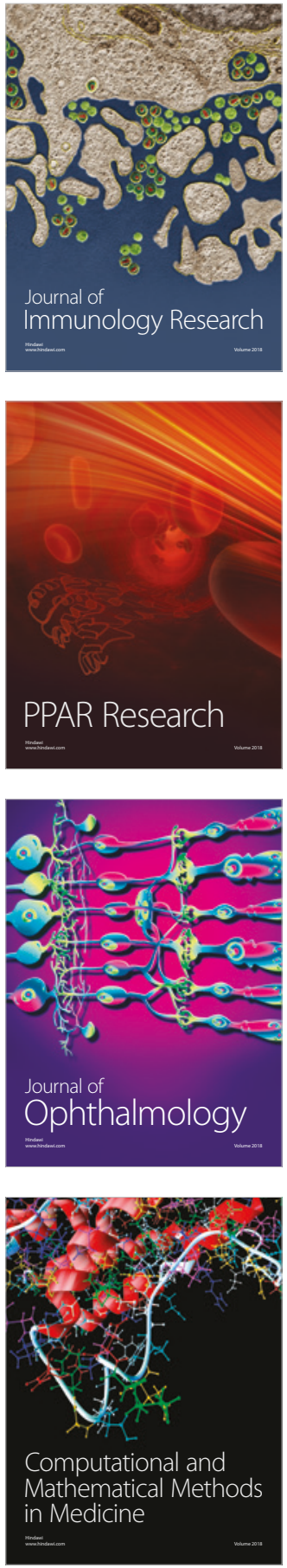

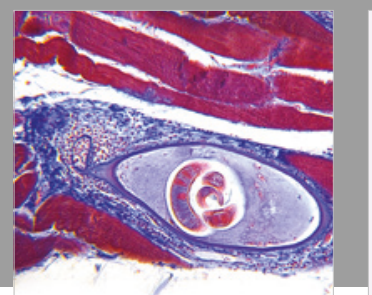

Gastroenterology Research and Practice

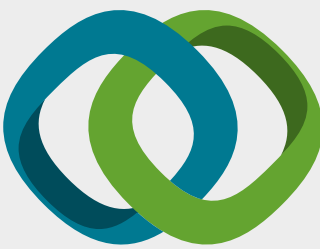

\section{Hindawi}

Submit your manuscripts at

www.hindawi.com
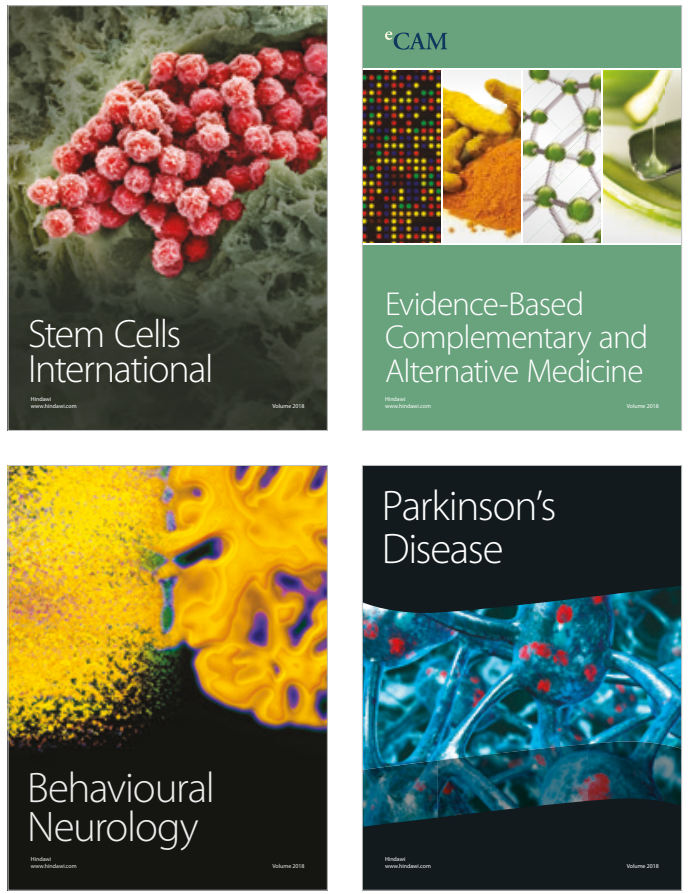

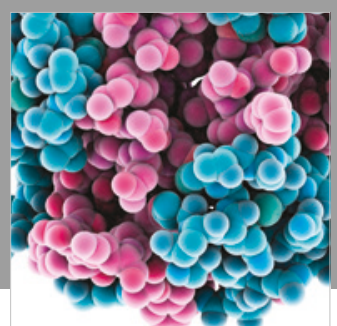

ournal of

Diabetes Research

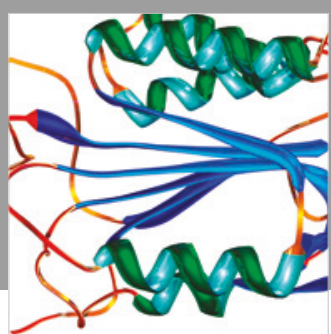

Disease Markers
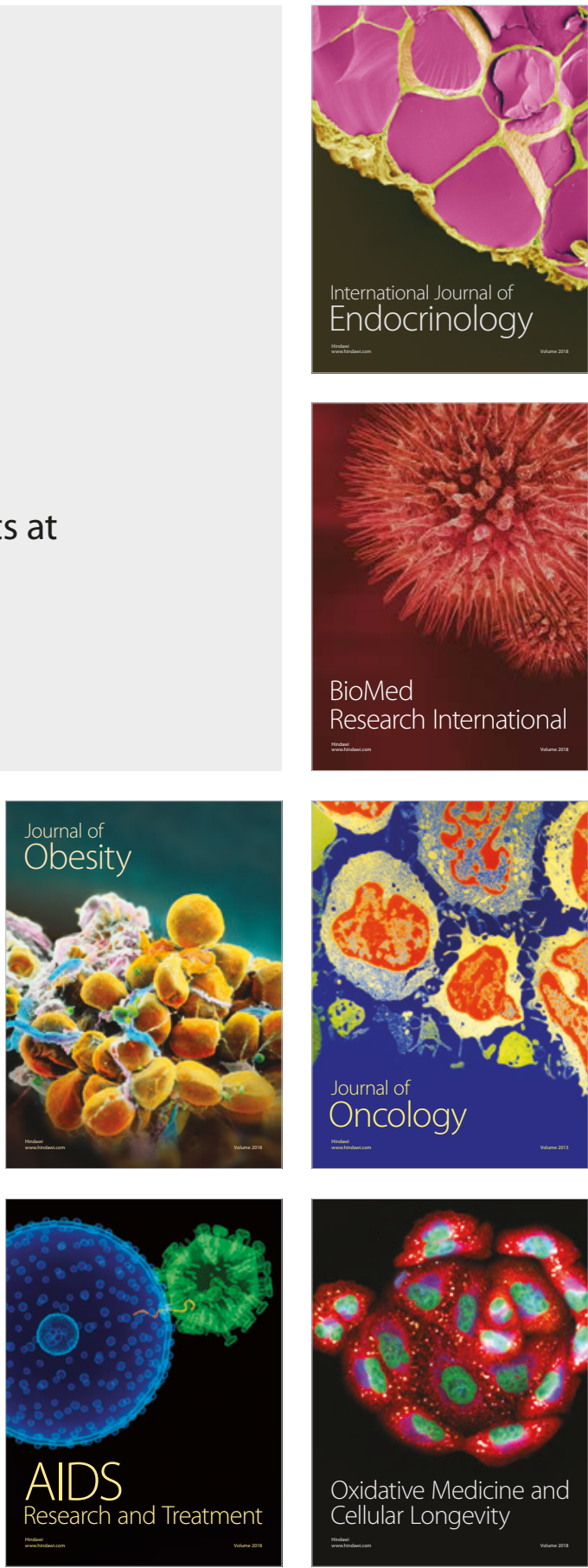